NP3 (continued)

improve dietary quality in low-income families by conducting research, extension, and education activities.

Description: The Farm Fresh Foods for Healthy Kids (F3HK) intervention included a cost-offset (50\% subsidy) summer CSA share, weekly payments (including SNAPEBT), two to four pieces of kitchen equipment, and nine CSA-tailored education classes. F3HK participants were recruited from 12 rural and micropolitan communities in four states in spring 2016 and 2017, and individually randomized to intervention $(n=148)$ or control $(n=157)$. All had income $<185 \%$ poverty and at least one child $(2-12 y)$ in the household. Using an intent-to-treat framework, multivariate multi-level regression models were used to examine change in outcomes over time for intervention relative to control. Geospatial data, participant focus groups, and farmer interviews were used to describe COCSA accessibility.

Evaluation: CO-CSA pickup sites averaged six miles (m) from participants' homes, which was closer than the farm $(18 \mathrm{~m})$ but further than the supermarket $(3 \mathrm{~m})$. F3HK farms reported efforts to reach low-income customers and strategized to modify the F3HK model to suit the local context; however, CO-CSA participants expressed mixed levels of accessibility. Relative to controls, F3HK intervention caregivers had improvement in some measures of attitudes, self-efficacy, skills, and dietary quality after one-season of participation, but not other measures. Lessons from the implementation and evaluation of F3HK informed the development of four undergraduate course modules: development of an intervention in a setting where "local food" is a foreign concept; assessment of dietary quality; economic impact analysis; and how to adapt CSA to open new markets for farmers.

Conclusion: CO-CSA plus education and kitchen tools is a promising mechanism to improve attitudes, self-efficacy, and skills among caregivers, with mixed results concerning dietary quality among caregivers and children. Longer-term outcome data will be examined for behavioral maintenance. An Extension toolkit for CO-CSA implementation is under development. Education modules are being piloted, and will be evaluated and refined before dissemination in 2020-21.

Funding: 2015-68001-23230.

\section{NP4 Improving Preschool Children's Food Knowledge and Food Choice Intentions: Results From a Childhood Obesity Preventive Intervention}

LoriFrancis,PhD, 1francis@psu.edu, The Pennsylvania State University, 219 Biobehavioral Health Bldg, University Park, PA 16802; Michelle Mannino, VMD, The Pennsylvania State University; Brandi Rollins, PhD, The Pennsylvania State University

Objective: To examine the impact of a large, preventive intervention on preschool children's food knowledge, and intentions to choose nutrient-dense foods for meals and snacks.
Description: A total of 29 classrooms across 14 predominantly low-income, rural childcare centers $(n=215$ children, $52 \%$ female, mean age $=4.1 \mathrm{y}$ ) were randomized into 1 of 16 conditions that differed based on whether a classroom received an intervention curriculum (Healthy Eating, Active Play and Self-Regulation) or parent/caregiver guidance. For the purposes of this study, we focus solely on the 11-week Healthy Eating (HE) curriculum that covered a number of topics, including: identifying "GO" and "WHOA" foods, food groups, increasing fruit and vegetable intake, and building a "healthy plate."

Evaluation: Measures were collected pre-post. To assess children's food knowledge, children were first asked to choose their favorite foods from a list of 30 foods and four beverages that vary in energy content. Children were then asked to choose foods for a "healthy lunch" using foods from the same list. A snack selection procedure was used to assess children's food preferences and ability to choose the healthier choice from a list of nine pairs of foods; each pair contained an energy-dense and nutrient-dense food. Children in the HE condition showed a slightly larger increase in their ability to correctly identify nutrientdense foods. Children in the HE condition showed an increase in nutrition knowledge, and competence in making nutrient-dense snack and meal choices. Children in the HE condition also showed more than a two-fold greater increase in their ability to identify nutrient-dense snack foods than children who were not in the HE condition. Data collection with more than 700 participants is ongoing; results including these additional participants will be shared.

Conclusion and Implications: Increasing children's food/nutrition knowledge and intentions to choose nutrient-dense over energy-dense foods may lead to the development of healthy dietary patterns that prevent the development of obesity in young children.

Funding: 2015-68001-23233.

\section{NP5 Salud Para Usted y Su Familia [Health for You and Your Family]: Integration of Research, Education and Extension to Promote Healthier Mexican-Heritage Families}

Joseph Sharkey, PhD, MPH, jrsharkey@sph.tamhsc.edu, Texas A\&M University School of Public Health, 1266 TAMU, College Station, TX 77843-1266; Renee Umstattd Meyer, PhD, MCHES, FAAHB, Baylor University; Cassandra Johnson, PhD, MSPH, Texas State University; Luis Gomez, $B A$, Texas A\&M University School of Public Health; Luz Martinez, $B A$, Texas A\&M University School of Public Health; Elva Beltran, $B A, C H W$, Texas A\&M University School of Public Health; Tomas Johanson, MPH, Texas A\&M University School of Public Health

Objective: To implement and evaluate an integrated Spanish-language program to improve nutrition, increase physical activity, and strengthen family function among Mexican-heritage (MH) families. We describe research (stepped wedge cluster randomized controlled trial 